\title{
BILATERAL DEFICIT: RELATIONSHIPS WITH TRAINING HISTORY AND FUNCTIONAL PERFORMANCE
}

\author{
Gareth Nicholson and Dario Masini \\ Carnegie School of Sport, Leeds Beckett University, UK
}

Original scientific paper

DOI: $10.26582 / \mathrm{k} .53 .1 .11$

\begin{abstract}
:
The purpose of the study was to investigate the magnitude of bilateral deficit (BLD) in trained males and examine its relationship with functional performance and recent resistance training history. Ten physically active males (age: $23.02 \pm 1.27$ years) self-reported the number of unilateral and bilateral exercises within their structured resistance training schedule. During two visits to the laboratory, participants performed unilateral and bilateral squat jumps (SJ) and isometric leg extensions (ILE) for the quantification of BLD. Participants also performed bilateral countermovement jumps (CMJ) and a change of direction (COD) test to quantify functional performance. The performance outcomes and information regarding training history were then correlated with the bilateral index (BLI) metric. The key findings were that: (a) a lower BLD in SJ peak power related to a greater CMJ peak force $(r=.728 ; p=.02)$ and peak power $(r=.750 ; p=.01)$, (b) the BLI in the ILE was unrelated to performance outcomes, and (c) BLI was unrelated to the mean number of bilateral and unilateral exercises in the structured resistance training programme of participants. In conclusion, lower levels of BLD may be advantageous for bilateral tests of functional performance (i.e. jumps), however there is a need to consider the mechanical similarity between the performance and BLD measure. Finally, the balance of unilateral and bilateral exercises in an individual's recent resistance training history is not sensitive to the BLI measured during dynamic or isometric assessments.
\end{abstract}

Key words: unilateral, strength, jump, specificity

\section{Introduction}

The bilateral limb deficit (BLD) phenomenon describes the deficit in force generating capacity when bilateral contraction is less than the combined force production of right and left limbs contracting alone (Janzen, Chilibeck, \& Davison, 2006; Kuruganti, Murphy, \& Pardy, 2011). The investigation of this phenomenon necessitates the performance of unilateral and bilateral trials of the same task (e.g. jumps). Several theories have been put forward to explain the mechanisms underpinning BLD; these include neuromuscular factors relating to motor unit recruitment (Koh, Grabiner, \& Clough, 1993) and other neural factors (Howard \& Enoka, 1991), mechanical factors relating to the force-velocity relationship (Bobbert, Graaf, Jonk, \& Casius, 2006) and methodological issues relating to counterbalancing unilateral and bilateral tasks (Magnus \& Farthing, 2008; Simoneau-Buessinger, et al., 2015). Although the debate persists regarding the predominant mechanisms underpinning BLD, this phenomenon is known to occur in young and old individuals (Kuruganti, Parker, Rickards, Tingley, \& Sexsmith, 2005), males and females (Škarabot, Cronin, Stroj- nik, \& Avela, 2016), and across differing muscle groups and actions (Kuruganti \& Seasman, 2006).

From an applied perspective, it has been theorised that the ability to express force unilaterally or bilaterally can affect athletic performance depending on the limb involvement of the predominant sporting movements (Škarabot, et al., 2016). Whilst this remains an intriguing concept for the practitioner, the association between BLD and athletic performance has only recently been explored. Bračič, Supej, Peharec, Bačić, and Čoh (2010) demonstrated that a smaller BLD (measured during the countermovement jump) was associated with a higher peak force production $(\mathrm{r}=-.63, \mathrm{p}<.01)$ and higher total impulse $(\mathrm{r}=-.55, \mathrm{p}<.01)$ during block starts in sprinters adding credence to the suggestion that a BLD may not be advantageous for athletes in sports (e.g. rowing) that require predominately bilateral movements (simultaneous action of both limbs). More recently, Bishop et al., (2019) reported that BLD is associated with faster change of direction (COD) speed when quantified via the countermovement jump and drop jump metrics highlighting that BLD may be desirable in sports (e.g. team sports) requiring frequent unilateral actions 
(e.g. kicking, COD). Furthermore, this study highlights that BLD is associated with performance outcomes (e.g. COD's time) and not just underpinning mechanical variables (e.g. impulse) as in the investigation by Bračič et al., (2010).

Whilst these investigations underline that the magnitude and existence of BLD may be of importance for athletes and practitioners attempting to maximise physical performance, these studies utilised only jumping assessments of BLD. Examination of literature reveals a range of tests used to assess BLD (Škarabot, et al., 2016), with BLD being demonstrated during isometric (Howard \& Enoka, 1991; Magnus \& Farthing, 2008; Botton, et al., 2013), isokinetic (Brown, Whitehurst, Gilbert, Findley, \& Buchalter, 1994; Dickin \& Too, 2006), and explosive (Buckthorpe, Pain, \& Folland, 2013; Challis, 1998; Pain, 2014) tasks. Furthermore, a large number (e.g. Koh, et al., 1993; Howard \& Enoka, 2006; Botton, et al., 2013) have used singlejoint strength assessments such as the isometric knee extension. Whilst these types of assessments can assist in controlling important methodological factors (e.g. counterbalancing, muscle recruitment) and further investigation of potential underlying mechanisms, the mechanical similarity of multijoint BLD assessments (e.g. jumps) may have a greater transferability to sporting applications and thus stronger associations with physical performance.

Further support for the link between BLD and physical performance can be gained from the fact that athletes of different disciplines have been shown (Howard \& Enoka, 1991) to exhibit differing levels of BLD, with some athletes also demonstrating bilateral limb facilitation (BLF) (i.e. maximal bilateral force production is greater than the sum of unilateral forces). More specifically, Howard and Enoka (1991) previously reported that cyclists demonstrated greater levels of BLD (the bilateral force production $6.6 \pm 7.1 \%$ less than the combined unilateral) when compared to Olympic weightlifters (the bilateral force production $6.2 \pm 4.7 \%$ greater than the combined unilateral) (Howard \& Enoka, 1991). This has led to the theory that training history and previous exposure to exercise may exacerbate levels of BLD (Janzen, et al. 2006). In support of this, more recent studies have demonstrated that unilateral and bilateral resistance training routines can either augment or attenuate bilateral force production in athletes (Botton, et al., 2016; Janzen, et al. 2006). Whilst the trainability of BLD is an attractive proposition for the practitioner given the proposed links with physical performance, it is not clear whether the balance of unilateral and bilateral exercises in an individual's recent resistance training programme is reflective of the magnitude or existence of BLD. Such a concept would enable practitioners to evaluate the extent to which bilateral and unilateral exercise selection is optimal for the performance outcomes of a given sport.

While previous research has established that $\mathrm{BLD} / \mathrm{BLF}$ is related to measures of physical performance (Bishop, et al., 2019; Bračič, et al., 2010), the influence of mechanical similarity (i.e. singleand multi-joint assessments) on this relationship has not been well explored. Furthermore, while research has alluded to differences in BLD and BLF in athletes of differing sports (Howard \& Enoka, 1991) and training histories (Janzen, et al., 2006), the associations with exercise selection have not been well established. Consequently, the aims of this study were to: 1) investigate the relationships between BLD/BLF across both single- and multijoint tests and physical performance; and 2) assess the relationship between individual BLD/BLF and recent training history.

\section{METHODS}

\section{Study design}

To assess the relationship between measures of single- and multi-joint BLD/BLF in the lower limbs, and physical performance and training history, 10 participants were recruited and completed a range of isometric and dynamic assessments across two days of testing.

Prior to the commencement of the study, participants were provided with instructions, allowed to ask questions and afforded the opportunity to practice each assessment to reduce any learning effects during the study (Hopkins, 2000). Upon arrival at the laboratory on the first day of testing, participants completed a training history questionnaire that detailed frequency of unilateral and bilateral resistance training, followed by unilateral and bilateral trials (randomised order) of the squat jump (SJ) and isometric leg extension (ILE) to quantify bilateral index. On the second day, participants returned to the same laboratory and completed bilateral trials of the countermovement jump (CMJ) and a change of direction (COD) test to quantify physical performance (also in a randomised order). Testing days were separated by 48 hours and participants were asked to refrain from vigorous exercise and maintain a consistent diet for the duration of the study. The best trial was used for subsequent analysis for the ILE (trial with the highest peak force) and jump (trial with the greatest jump height during the SJ and $\mathrm{CMJ}$ ) assessments.

\section{Participants}

Ten recreationally trained males (age: $23 \pm 1.3$ years; body height: $1.80 \pm 0.54 \mathrm{~m}$; body mass: $82.6 \pm 12.4 \mathrm{~kg}$ ) provided written consent to participate in this study. To be eligible for participation, participants were required to be aged 18 years, 
free from injury, had at least two years resistance training experience (Weakley, et al., 2017a) and trained the lower-body at least two times per week. Additionally, participants regularly performed jumping assessments and did not take any medications that could affect the results of the tests. All experimental procedures were approved by the Leeds Beckett University's ethics committee and written consent was provided by all participants.

\section{Quantification of weekly unilateral and bilateral lower body exercises}

To assess the quantity of weekly unilateral and bilateral lower-body exercises, participants were asked "Approximately how many unilateral lowerbody exercises do you perform in your weekly resistance training programme?" and "Approximately how many bilateral lower-body exercises do you perform in your weekly resistance training programme?". Participants reported these frequencies to the nearest whole number and were instructed to base their responses on the four weeks of training preceding the commencement of the study.

\section{Procedures}

At the start of each testing session, participants completed a standardised warm-up which included five minutes of a low-intensity aerobic exercise (jogging at a self-selected speed), dynamic mobilisation exercises and submaximal repetitions (50 and $75 \%$ ) of the exercises to be completed during testing. A five-minute recovery was permitted in between the end of the warm-up and the commencement of data collection. On the first visit, the ILE was performed prior to the SJ assessment and on the second visit, the CMJ was performed prior to the COD assessment. The order of these tests (ILE $>$ SJ $>\mathrm{CMJ}>\mathrm{COD}$ ) was selected based upon previous recommendations provided by the National Strength and Conditioning Associate (NSCA) which had been shown to maximise the reliability of testing procedures (Miller, 2012). A three-minute recovery was permitted between each separate assessment.

\section{Measurement of bilateral deficit/facilitation}

To quantify the magnitude of BLD/BLF for each participant, three different variations (i.e. unilateral left, unilateral right and bilateral) of the SJ and ILE were used. A mono-articular and multiarticular test were chosen to provide a comprehensive overview of BLD/BFD and both had previously been used to assess asymmetry and BLD in males of a similar age and training level (Kuruganti \& Murphy, 2008; Yoshioka, Nagano, Hay, \& Fukashiro, 2011). Furthermore, as an exercise type may influence the results of BLD measures (Škarabot, et al., 2016), this study utilised both isometric and dynamic assessments.

\section{Squat jump}

The SJ was used due to its high levels of reliability and validity (Markovic, Dizdar, Jukic, \& Cardinale, 2004). It was selected over the CMJ for the assessment of BLD as it reduces the potential bias of completing the countermovement (eccentric) portion of the exercise and assists in the standardisation of the starting position (Kubo, Tsunoda, Kanehisa, \& Fukunaga, 2004). To ensure consistency between trials and participants, each participant was required to lower himself to a self-selected depth with which they chose to complete a unilateral SJ. The angle of the knee was recorded for each participant using a clinical goniometer $(\mathrm{M} \pm \mathrm{SD}$ : $114^{\circ} \pm 8$ ) and then standardised between trials. Knee flexion was maintained for two seconds prior to any vertical movement. Hands were required to remain on the hips throughout the three attempts of the three SJ variations (two unilateral and one bilateral) being completed in a randomised order. A one-minute rest was provided between maximal efforts, with participants being instructed to jump as high as possible and to maintain full lower-body extension during the flight phase of the jump. All squat jump attempts were performed on a force platform (Kistler 9287BA; Winterthur, Switzerland) sampling at $1,000 \mathrm{~Hz}$. BioWare software (Version 5.3.2.9.; Kistler Instrument Corp.) was used for data analysis. From the three attempts of each SJ variation, the attempt with the highest recorded flight time was kept and used for analysis. Jump height was obtained using the flight time method $(0.5 \mathrm{x}$ $9.81 \times$ [flight time $/ 2]^{2}$ ), along with peak vertical force, peak concentric power and average concentric power prior to take-off. Between-trial reproducibility for jump height was high for the bilateral $(\mathrm{ICC}=.92)$ and unilateral trials (ICC $=.91-.93)$.

\section{Isometric leg extension}

The maximal isometric force and explosive force generating characteristics of the knee extensor muscles were measured using a custommade isometric device consisting of a customised leg extension machine (GLCE365, Body Soild UK), which was connected to a force platform (Kistler 9253B22, 1,000 Hz) via a chain (Emmonds, Nicholson, Beggs, Jones, \& Bissas, 2017). Participants were seated on the leg extension machine with a trunk-thigh angle of $90^{\circ}$ with two crossover shoulder harnesses and a belt across the abdomen limiting all extraneous movements of the upper body (Matkowski, Place, Martin, \& Lepers, 2011). Due to the discrepancy in previous results of measuring BLD at different knee angles in the ILE (Škarabot, et al., 2016), the knee angle ( $\left.\mathrm{M} \pm \mathrm{SD}: 114^{\circ} \pm 8\right)$ utilised by each subject during the squat jump was comparable with the angle used previously in the BLD literature (Botton, et al. 2013). Participants were required to complete three submaximal familiar- 
isation contractions prior to data collection for each protocol (i.e. two unilateral and one bilateral) followed by three minutes of recovery. Following this, participants were then instructed to follow the countdown ('three, two, one-push') and exert as rapidly as possible maximal force maintaining it during a time period of five seconds. The participants were required to complete two maximum voluntary contractions for each ILE variation, in a randomised order. These different variations have previously been utilised for the calculation of BLD (Howard \& Enoka, 1991). Three-minute recovery periods were allowed between maximal attempts and the trial with the highest peak force was taken forward for further analysis. Force-time data was analysed using Bioware software (Version 5.3.2.9.; Kistler Instrument Corp.) with peak resultant force (N) and rate of force development (RFD) during the initial $250 \mathrm{~ms}$ of contraction being assessed. The between-trial reproducibility for peak force was high for both the bilateral $(\mathrm{ICC}=.97)$ and unilateral trials ( $\mathrm{ICC}=.93-.98)$.

\section{Measurement of physical performance}

To quantify physical performance, maximal trials of the CMJ and COD assessment were completed in the second testing session. These assessments were chosen based on their frequent use in training, testing and in competition.

\section{Countermovement jump}

The CMJ has previously been used to investigate the link between BLD/BLF and physical performance (Bračič, et al., 2010; Gonzalo-Skok, et al., 2017) and has been shown to be a reliable field test for the estimation of lower-body function (Markovic, et al., 2004). All maximal CMJ attempts were completed upon a force plate (9287BA/CA, 40 x $60 \mathrm{~cm}$, Kistler, Winterthur, Switzerland) sampling at $1,000 \mathrm{~Hz}$. CMJs were performed with a preparatory downward movement following an upright starting position with the feet placed approximately shoulder width apart. Participants lowered themselves to a self-selected depth and jumped as high as possible. Between each maximal exertion, a oneminute rest was provided (Weakley, et al., 2019a,b). Out of the three attempts, the CMJ with the greatest flight time was kept for analysis and jump height (calculated using flight time), peak vertical force, peak concentric power and mean concentric power were calculated. Subjects were instructed to jump as "high as possible" while maintaining their hands upon their hips at all times (Sawczuk, et al., 2018; Weakley, et al., 2019b). The between-trial reproducibility for jump height was high (ICC=.97).

\section{Change of direction test}

The COD assessment consisted of a 5-m sprint in a straight line, followed by a $135^{\circ}$ change of direction and subsequent 5-m acceleration (Sheppard \& Young, 2006). Two sets of timing gates (Witty Microgate, Italy) were positioned at the beginning and end point to assess total test time (recorded to the nearest $0.01 \mathrm{~s}$ ). Participants were required to start 0.5 metres behind the first timing gates and accelerate with maximal effort. When assessing the left leg, participants were asked to touch a marker at the 5-m point, change direction with the left leg $\left(135^{\circ}\right.$ to the right) then sprint further five metres through the final timing gates. The turning leg was alternated on each repetition to assess differences between the lower limbs. Each limb was tested three times with a one-minute recovery between the attempts. The between-trial reproducibility for the left $(\mathrm{ICC}=.94)$ and right $(\mathrm{ICC}=.97)$ limbs was high.

\section{Statistical analysis}

Statistical analysis was performed using SPSS version 24.0 for Windows (Chicago, IL, USA). The best performance for each test was chosen for statistical processing and normality was inspected using the Shapiro-Wilk test. The within-trial reliability for each assessment was assessed using an intraclass correlation coefficient (ICC) with absolute agreement $(3,1)$. The bilateral index of all SJ and ILE metrics were calculated using the same method as previously outlined (Howard \& Enoka, 1991):

$$
\begin{gathered}
B I(\%)=\{100[\text { bilateral } / \\
(\text { right unilateral }+ \text { left unilateral })]\}-100 .
\end{gathered}
$$

A negative bilateral index indicates that the value of the two-legged variation is smaller than the sum of the two unilateral variations (i.e. a BLD). Pearson correlation coefficients were used to determine the relationship between the BLI for the SJ (jump height, peak power, peak force) and ILE metrics (peak force, RFD) with the performance metrics obtained from the CMJ (jump height, peak power, peak force, average power) and COD (time) assessments. To determine the association between BLD/BLF and training history, Pearson's correlations were used to quantify the relationship between the number of unilateral / bilateral lower-body exercises (collected through the questionnaire) and the BLI. Statistical significance was set at $\mathrm{p}<.05$.

\section{Results}

All data was deemed normally distributed. Mean scores for the unilateral and bilateral assessments used to quantify BLI and physical performance are presented in Table 1. 
Table 1. Mean (SD) test data for the bilateral and unilateral tests

\begin{tabular}{lccc}
\hline Test (metric) & Both & Left & Right \\
\hline ILE PF (N) & $1217.60(410.89)$ & $545.17(207.20)$ & $591.62(191.39)$ \\
ILE RFD (N/s) & $3174.13(1155.04)$ & $1477.25(564.36)$ & $1522.44(564.56)$ \\
SJ JH (m) & $0.36(0.05)$ & $0.18(0.03)$ & $0.19(0.03)$ \\
SJ PP (W) & $4870.95(843.71)$ & $2637.44(387.38)$ & $2811.93(359.22)$ \\
SJ PF (N) & $2367.94(306.70)$ & $1605.04(200.51)$ & $1644.82(181.18)$ \\
SJ AP (W) & $2437.66(410.66)$ & $1094.24(163.09)$ & $1156.48(188.53)$ \\
CMJ JH (m) & $0.45(0.06)$ & & \\
CMJ PP (W) & $4791.50(837.74)$ & & \\
CMJ PF (N) & $2073.56(327.59)$ & & \\
CMJ AP (W) & $2669.34(426.46)$ & & $2.52(0.14)$ \\
COD (s) & & $2.54(0.14)$ & \\
\hline
\end{tabular}

Note. ILE = isometric leg extension, $\mathrm{PF}=$ peak force, $\mathrm{RFD}=$ rate of force development, $\mathrm{SJ}=$ squat jump, $\mathrm{JH}=$ jump height, $\mathrm{PP}=$ peak power, $\mathrm{AP}=$ average power, $\mathrm{CMJ}=$ countermovement jump, $\mathrm{COD}=$ change of direction.

Figure 1 illustrates the BLI for the ILE and SJ assessments with the ILE metrics showing a BLF when averaged across all participants and the SJ metrics showing a BLD when averaged across participants.

Pearson's $r$ correlations between each BLI metric and the variables measured during the performance tests are presented in Table 2. A significant position correlation was observed between the SJ peak power output and CMJ peak power $(\mathrm{r}=.750$, $\mathrm{p}<.05)$, CMJ peak force $(\mathrm{r}=.728, \mathrm{p}<.05)$ and $\mathrm{CMJ}$ average power $(\mathrm{r}=.802, \mathrm{p}<.01)$.

Table 3 displays the Pearson's $r$ correlations between the self-reported frequency of unilateral and bilateral resistance training exercises and the

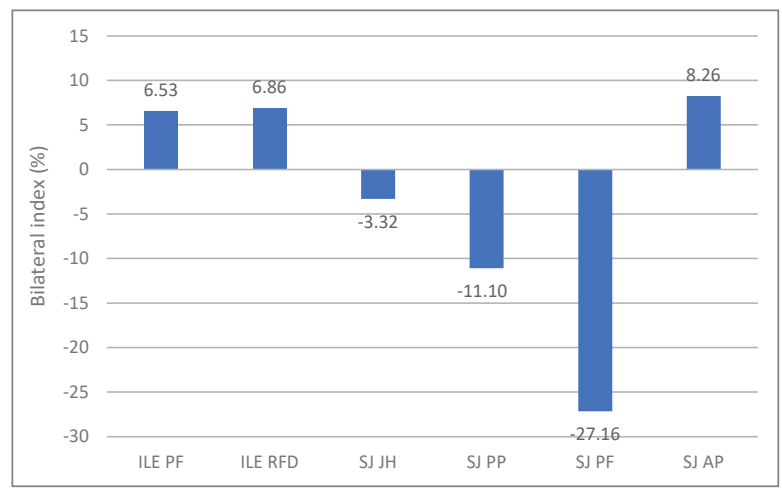

Figure 1. Bilateral index for the isokinetic knee extension (IKE) metrics and squat jump (SJ) metrics. $P F=$ peak force, $R F D=$ rate of force development, $J H=$ jump height, $P P=$ peak power, $A P=$ average power.

Table 2. Pearson's $r$ correlations between bilateral index \% scores and the jumping and agility performance measures

\begin{tabular}{lcccccc}
\hline $\begin{array}{l}\text { Bilateral Index } \\
(\%)\end{array}$ & $\begin{array}{c}\text { CMJ } \\
\text { JH }\end{array}$ & $\begin{array}{c}\text { CMJ } \\
\text { PP }\end{array}$ & $\begin{array}{c}\text { CMJ } \\
\text { PF }\end{array}$ & $\begin{array}{c}\text { CMJ } \\
\text { AP }\end{array}$ & $\begin{array}{c}\text { COD } \\
\text { (Left) }\end{array}$ & $\begin{array}{c}\text { COD } \\
\text { (Right) }\end{array}$ \\
\hline ILE PF & -.467 & .201 & .140 & .025 & .198 & .069 \\
ILE RFD & .083 & .480 & .353 & .397 & -.270 & -.321 \\
SJ JH & .318 & .081 & .066 & .103 & .454 & .581 \\
SJ PP & .350 & $.750^{*}$ & $.728^{*}$ & $.802^{* *}$ & -.228 & -.219 \\
SJ PF & .190 & .006 & .044 & .111 & -.360 & -.299 \\
SJ AP & .230 & .187 & .318 & .293 & .079 & .190 \\
\hline
\end{tabular}

Note. ILE = isometric leg extension, $\mathrm{PF}=$ peak force, $\mathrm{RFD}=$ rate of force development, $\mathrm{SJ}=$ squat jump, $\mathrm{JH}=$ jump height, $\mathrm{PP}=$ peak power, $\mathrm{AP}=$ average power, $\mathrm{CMJ}=$ countermovement jump, $\mathrm{COD}=$ change of direction.

${ }^{*}$ correlation is significant at $p<.05$

${ }^{* *}$ correlation is significant at $p<.01$

Table 3. Pearson's $r$ correlations between the number of unilateral/bilateral exercises performed in participants' weekly training routine and the bilateral index obtained for the squat jump and isometric leg extension metrics

\begin{tabular}{lcccccc}
\hline Bilateral Index (\%) & SJ JH & SJ PP & SJ PF & SJ AP & IKE PF & IKE RFD \\
\hline No. of unilateral exercises & -0.26 & -0.487 & -0.339 & -0.137 & 0.451 & -0.343 \\
No. of bilateral exercises & -0.201 & 0.127 & 0.198 & -0.389 & 0.318 & 0.320 \\
\hline
\end{tabular}

Note. IKE = isokinetic knee extension, $\mathrm{PF}=$ peak force, $\mathrm{RFD}=$ rate of force development, $\mathrm{SJ}=$ squat jump, JH = jump height, $\mathrm{PP}=$ peak power, AP = average power.

${ }^{*}$ correlation is significant at $p<.05$

${ }^{* *}$ correlation is significant at $p<.01$ 
bilateral index of the metrics obtained during the SJ and ILE. The mean $( \pm \mathrm{SD})$ number of unilateral and bilateral exercises completed per week reported by the subjects were $2.6 \pm 1.84$ and $6.4 \pm 2.32$, respectively.

\section{Discussion and conclusion}

The aims of this study were: 1) to investigate the possible correlation between BLD/BLF across both the single- and multi-joint tests (i.e. SJ and ILE) and the physical performance measures (i.e. CMJ and COD); and 2) to assess the relationship between individual BLD/BLF and previous resistance training history. Findings demonstrated a very high correlation between the peak power bilateral index of the SJ and CMJ peak force $(\mathrm{r}=.728$; $\mathrm{p}=.017)$, CMJ peak power $(\mathrm{r}=.750 ; \mathrm{p}=.012)$, and CMJ mean power $(r=.802 ; p=.005)$, which suggests that the BLD should be an important consideration for the development of force and power production in bilateral jumping movements. Vast differences in the magnitude and direction (i.e. BLF/BLD) of the bilateral index were observed between the singleand multi-joint assessments and their associated metrics highlighting the need to consider mechanical similarity when measuring and interpreting BLD. Finally, the self-reported mean number of bilateral and unilateral exercises performed in the usual routine of the participants, was not shown to be significantly correlated with the BLD levels (Table 3).

Research has suggested that BLD/BLF may be of importance for physical performance (Bračič, et al., 2010; Bishop, et al., 2019). The current study supports this tenet, with increases in peak power bilateral index in the $\mathrm{SJ}$ showing very large $(\mathrm{r}=.73$ to .80 ) relationships with peak force, peak power and mean power in the bilateral CMJ. Previously, Bračič et al. (2010) showed significant correlations between bilateral index in peak force production during the CMJ and the peak force and impulse produced in the double sprint start. These results agree with the findings of our study and highlight that a reduction in BLD (i.e. an increase in bilateral index) may enhance kinetic and kinematic outputs in bilateral tasks. Thus athletes and practitioners may wish to consider interventions which promote BLF in peak power when training for sports which predominantly involve movements of the lowerbody (e.g. Olympic weightlifting). Since the association was confined to the underpinning SJ mechanical variables (i.e. peak power) and not the performance outcome (i.e. SJ jump height), the need to conduct an in-depth analysis of this phenomenon is highlighted.

A key finding was the failure to observe significant relationships between bilateral index and COD performance. Previous studies have also failed to observe relationships between bilateral index and unilateral performance measures (i.e. sprint times), however Bishop et al. (2019) recently observed significant negative correlations between BLD and COD speed. Considering the paucity of research examining the association between BLD and performance, conclusive interpretation remains difficult. Nevertheless, it should be noted that the previous study utilised a test of COD speed (i.e. 505 agility) that is different to that of the current study. Furthermore, it is apparent that COD tasks involve horizontal and vertical force production and necessitate braking and propulsive strategies which most likely involve eccentric and concentric muscle actions of the lower musculature. Since the previous study utilised a CMJ as a measure of BLD, it seems plausible that the concentric nature of the SJ used in the present study may have contributed to the lack of association with COD performance. Whilst such a suggestion remains speculative, practitioners and scientists should be mindful of the mechanical characteristics of different tasks when investigating the link between BLD and performance.

Although recent studies have reported associations between BLD measured during jump tasks and physical performance (Bračič, et al., 2010; Bishop, et al., 2019), this is the first study to examine these associations using alternative assessments of BLD. Indeed, this study observed no significant associations between $\mathrm{CMJ}$ or COD performance and BLI for any metric in the ILE. The bilateral index has previously shown great variability when measured using the isometric leg extension (Škarabot, et al., 2016), as such the BLF observed in isometric PF and RFD may not have been unexpected. The present findings however go one step further by highlighting that single-joint isometric assessments of BLD may not show strong associations with dynamic performance outcomes. Indeed, previous research has shown greater relationships between dynamic tests of physical performance when compared to isometric assessments, and this appears to be consistent when assessing BLD (Nuzzo, Mcbride, Cormie, \& McCaulley, 2008).

In terms of the magnitude of the BLI, the BLD observed for peak force $(-26.65 \% \pm 4.84)$ and jump height $(-1.95 \% \pm 12.08)$ in the SJ (Table 2) were noticeably less than those found in the CMJ by Bračič et al. (2010) (33.9 and 19.1\%, respectively) and Bishop et al., (2019) (21.76 and 12.67\%, respectively). Although differences in population should be considered, the greater BLI (particularly in jump height) may be due to differences in standardisation of range of motion in the SJ and CMJ. In the study by Bračič et al. (2010), the range of motion in the unilateral CMJ was less than in the bilateral (mean difference in the knees: $\sim 18 \%$ and hips $\sim 12 \%$ ), whilst in the present study the range of motion in the SJ was standardised for both bilateral and unilateral variations. This suggests that 
when attempting to measure BLD, sport scientists and practitioners may need to consider the test and standardisation method that occurs. In particular, differences in jump strategy may augment or reduce discrepancies that may alter performance outcomes.

The fact that a mean BLF was observed for peak force $(6.53 \pm 7.06 \%)$ and rate of force development $(6.86 \pm 16.85 \%)$ in the ILE may have been expected since individuals reported a greater frequency of bilateral than unilateral exercises in their recent resistance training programme. However, the reported mean number of unilateral and bilateral resistance training exercises performed each week were found to have small to moderate relationships with individual bilateral index. Instead, it is important to point out that there was clear variation in the magnitude and occurrence of BLD/BLF between individuals and between assessments and the associated metrics. Recent research (Bishop, et al., 2021) into inter-limb asymmetries has highlighted a similar variation between tests and metrics, and whilst the findings of Bishop et al. (2019) corroborate this variation, the present findings demonstrate that this extends to isometric assessments of BLD. Consequently, scientists and practitioners are advised to carefully select the exercise and metric with which the bilateral index is assessed.

Previous research has suggested that training history may effect levels of BLD/BLF (Howard \& Enoka, 1991; McCurdy, Langford, Doscher, Wiley, \& Mallard, 2005), however the self-reported measures used in the current study did not show significant relationships. Several reasons may underlie this including the self-reported nature of the survey, the timescale over which the questions referred and the mechanical similarity between training and testing. Indeed, it is plausible that the exercises selected by participants did not resemble the tests used for the assessment of bilateral index (i.e. SJ and ILE). Early work by Schantz, Moritani, Karlson, Johansson, and Lundh (1989) found that changes in BLD might be masked if the testing exercise did not closely resemble the movement patterns of the previous resistance training. Thus, future research may be warranted to investigate the number of reported exercises that are directly related to the bilateral index tests performed (e.g. squat and CMJ).
While this study is the first to compare the relationships between physical performance, selfreported resistance training history and a selection of single- and multi-joint assessments of BLD, it is not without its limitations. Firstly, caution should be taken when interpreting correlational analyses with small sample sizes and therefore future BLD studies should seek to utilise a larger cohort when looking to explore the lack of association between BLI, physical performance and recent resistance training history. Second, while links between training history and individual BLD/BLF have previously been postulated (Howard \& Enoka, 1991; McCurdy, et al., 2005), the questionnaire provided to subjects within this study was unable to be adequately related to variances in subject bilateral index values. While this may be due to a range of reasons, it could be that the questionnaire provided was not sensitive to the many acute variables that underlie resistance training and poor validity of subject recollection of training history (Phibbs, et al., 2017). Future research may wish to more closely examine previous training history and assess whether more objective training data (e.g. training programmes and volume) are associated with differences in bilateral index.

This study presents and compares the relationship between measures of bilateral index and physical performance. Furthermore, the relationship between self-reported lower body resistance training history and bilateral index were investigated. Findings suggest that lower levels of BLD are related to greater force and power output in bilateral exercise. This may be of particular interest for coaches and athletes who participate in sports that complete predominantly bilateral movements (e.g. weightlifting, rowing). In contrast, isometric tests of bilateral index may poorly relate to dynamic performance underlining the need to consider the mechanical similarities between the performance and BLD measures. Finally, self-reported lower limb resistance training frequency was poorly related to BLD/ BLF. This may be due to training specificity or discrepancies in subject recollection. 


\section{References}

Bishop, C., Berney, J., Lake, J., Loturco, I., Blagrove, R., Turner, A., \& Read, P. (2019). Bilateral deficit during jumping tasks: Relationship with speed and change of direction speed performance. Journal of Strength and Conditioning Research, publish ahead of print.

Bishop, C., Lake, J., Loturco, I., Papadopoulos, K., Turner, A., \& Read, P. (2021). Interlimb asymmetries: The need for an individual approach to data analysis. Journal of Strength and Conditioning Research, 35(3), 695-701.

Bobbert, M.F., de Graaf, W.W., Jonk, J.N., \& Casius, L.R. (2006). Explanation of the bilateral deficit in human vertical squat jumping. Journal of Applied Physiology, 100(2), 493-499.

Botton, C.E., Radaelli, R., Wilhelm, E.N., Rech, A., Brown, L.E., \& Pinto, R.S. (2016). Neuromuscular adaptations to unilateral vs. bilateral strength training in women. Journal of Strength and Conditioning Research, 30(7), 1924-1932.

Botton, C.E., Radaelli, R., Wilhelm, E.N., Silva, B.G., Brown, L.E., \& Pinto, R.S. (2013). Bilateral deficit between concentric and isometric muscle actions. Isokinetics and Exercise Science, 21(2), 161-165.

Bračič, M., Supej, M., Peharec, S., Bačić, P., \& Čoh, M. (2010). An investigation of the influence of bilateral deficit on the counter-movement jump performance in elite sprinters. Kinesiology, 42(1), 73-81.

Brown, L.E., Whitehurst, M., Gilbert, R., Findley, B.W., \& Buchalter, D.N. (1994). Effect of velocity on the bilateral deficit during dynamic knee extension and flexion exercise in females. Isokinetics and Exercise Science, 4(4), 153-156.

Buckthorpe, M.W., Pain, M.T., \& Folland, J.P. (2013). Bilateral deficit in explosive force production is not caused by changes in agonist neural drive. PloS One, 8(3), e57549.

Challis, J.H. (1998). An investigation of the influence of bi-lateral deficit on human jumping. Human Movement Science, 17(3), 307-325.

Dickin, C.D., \& Too, D. (2006). Effects of movement velocity and maximal concentric and eccentric actions on the bilateral deficit. Research Quarterly for Exercise and Sport, 77(3), 296-303.

Emmonds, S., Nicholson, G., Beggs, C., Jones, B., \& Bissas, A. (2017). Importance of physical qualities for speed and change of direction ability in elite female soccer players. Journal of Strength and Conditioning Research, 33(6), 1669-1677.

Gonzalo-Skok, O., Tous-Fajardo, J., Suarez-Arrones, L., Arjol-Serrano, J.L., Casajús, J.A., \& Mendez-Villanueva, A. (2017). Single-leg power output and between-limbs imbalances in team-sport players: Unilateral versus bilateral combined resistance training. International Journal of Sports Physiology and Performance, 12(1), 106-114.

Hopkins, W.G. (2000). Measures of reliability in sports medicine and science. Sports Medicine, 30(1), 1-15.

Howard, J.D., \& Enoka, R.M. (1991). Maximum bilateral contractions are modified by neurally mediated interlimb effects. Journal of Applied Physiology, 70(1), 306-316.

Janzen, C.L., Chilibeck, P.D., \& Davison, K.S. (2006). The effect of unilateral and bilateral strength training on the bilateral deficit and lean tissue mass in post-menopausal women. European Journal of Applied Physiology, 97(3), 253-260.

Koh, T.J., Grabiner, M.D., \& Clough, C.A. (1993). Bilateral deficit is larger for step than for ramp isometric contractions. Journal of Applied Physiology, 74(3), 1200-1205.

Kubo, K., Tsunoda, N., Kanehisa, H., \& Fukunaga, T. (2004). Activation of agonist and antagonist muscles at different joint angles during maximal isometric efforts. European Journal of Applied Physiology, 91(2-3), 349-352.

Kuruganti, U., \& Murphy, T. (2008). Bilateral deficit expressions and myoelectric signal activity during submaximal and maximal isometric knee extensions in young, athletic males. European Journal of Applied Physiology, 102(6), 721-726.

Kuruganti, U., Murphy, T., \& Pardy, T. (2011). Bilateral deficit phenomenon and the role of antagonist muscle activity during maximal isometric knee extensions in young, athletic men. European Journal of Applied Physiology, 111(7), 1533-1539.

Kuruganti, U., Parker, P., Rickards, J., Tingley, M., \& Sexsmith, J. (2005). Bilateral isokinetic training reduces the bilateral leg strength deficit for both old and young adults. European Journal of Applied Physiology, 94(1-2), 175-179.

Kuruganti, U., \& Seaman, K. (2006). The bilateral leg strength deficit is present in old, young and adolescent females during isokinetic knee extension and flexion. European Journal of Applied Physiology, 97(3), 322-326.

Magnus, C.R., \& Farthing, J.P. (2008). Greater bilateral deficit in leg press than in handgrip exercise might be linked to differences in postural stability requirements. Applied Physiology, Nutrition, and Metabolism, 33(6), 1132-1139.

Markovic, G., Dizdar, D., Jukic, I., \& Cardinale, M. (2004). Reliability and factorial validity of squat and countermovement jump tests. Journal of Strength and Conditioning Research, 18(3), 551-555.

Matkowski, B., Place, N., Martin, A., \& Lepers, R. (2011). Neuromuscular fatigue differs following unilateral vs bilateral sustained submaximal contractions. Scandinavian Journal of Medicine and Science in Sports, 21(2), 268-276.

McCurdy, K.W., Langford, G.A., Doscher, M.W., Wiley, L.P., \& Mallard, K.G. (2005). The effects of short-term unilateral and bilateral lower-body resistance training on measures of strength and power. Journal of Strength and Conditioning Research, 19(1), 9-15. 
Miller, T.A. (2012). NSCA's Guide to Tests and Assessments. Human Kinetics.

Nuzzo, J.L., McBride, J.M., Cormie, P., \& McCaulley, G.O. (2008). Relationship between countermovement jump performance and multijoint isometric and dynamic tests of strength. Journal of Strength and Conditioning Research, 22(3), 699-707.

Pain, M.T. (2014). Considerations for single and double leg drop jumps: Bilateral deficit, standardizing drop height, and equalizing training load. Journal of Applied Biomechanics, 30(6), 722-727.

Phibbs, P.J., Roe, G., Jones, B., Read, D.B., Weakley, J., Darrall-Jones, J., \& Till, K. (2017). Validity of daily and weekly self-reported training load measures in adolescent athletes. Journal of Strength and Conditioning Research, 31(4), 1121-1126.

Sawczuk, T., Jones, B., Scantlebury, S., Weakley, J., Read, D., Costello, N., ..., \& Till, K. (2018). Between-day reliability and usefulness of a fitness testing battery in youth sport athletes: Reference data for practitioners. Measurement in Physical Education and Exercise Science, 22(1), 11-18.

Schantz, P.G., Moritani, T., Karlson, E., Johansson, E., \& Lundh, A. (1989). Maximal voluntary force of bilateral and unilateral leg extension. Acta Physiologica Scandinavica, 136(2), 185-192.

Sheppard, J.M., \& Young, W.B. (2006). Agility literature review: Classifications, training and testing. Journal of Sports Sciences, 24(9), 919-932.

Simoneau-Buessinger, E., Leteneur, S., Toumi, A., Dessurne, A., Gabrielli, F., Barbier, F., \& Jakobi, J.M. (2015). Bilateral strength deficit is not neural in origin; rather due to dynamometer mechanical configuration. PloS One, 10(12), e0145077.

Škarabot, J., Cronin, N., Strojnik, V., \& Avela, J. (2016). Bilateral deficit in maximal force production. European Journal of Applied Physiology, 116(11-12), 2057-2084.

Weakley, J.J., Till, K., Darrall-Jones, J., Roe, G.A., Phibbs, P.J., Read, D.B., \& Jones, B.L. (2017a). The influence of resistance training experience on the between-day reliability of commonly used strength measures in male youth athletes. Journal of Strength and Conditioning Research, 31(7), 2005-2010.

Weakley, J.J., Till, K., Read, D.B., Roe, G.A., Darrall-Jones, J., Phibbs, P.J., \& Jones, B. (2017b). The effects of traditional, superset, and tri-set resistance training structures on perceived intensity and physiological responses. European Journal of Applied Physiology, 117(9), 1877-1889.

Weakley, J., Till, K., Roe, G., Darrall-Jones, J., Phibbs, P., Read, D., \& Jones, B. (2019a). Strength and conditioning practices in adolescent rugby players: Relationship with changes in physical qualities. Journal of Strength and Conditioning Research, 33(9), 2361-2369.

Weakley, J., Till, K., Sampson, J., Banyard, H., Leduc, C., Wilson, K., Roe, G., \& Jones, B. (2019b). The effects of augmented feedback on sprint, jump, and strength adaptations in rugby union players following a four week training programme. International Journal of Sports Physiology and Performance, 14(9), 1205-1211.

Yoshioka, S., Nagano, A., Hay, D.C., \& Fukashiro, S. (2011). The effect of bilateral asymmetry of muscle strength on the height of a squat jump: A computer simulation study. Journal of Sports Sciences, 29(8), 867-877.

Submitted: July 26, 2019

Accepted: March 30, 2021

Published Online First: April 27, 2021

Correspondence to:

Gareth Nicholson, Ph.D.

Course Director

Room 107, Fairfax Hall

Headingley Campus

Leeds Beckett University

Leeds, LS6 3QT, UK

Email: g.nicholson@leedsbeckett.ac.uk 